\title{
Early-childhood housing mobility and subsequent PTSD in adolescence: a Moving to Opportunity reanalysis [version 1;
} peer review: 2 approved]

\author{
David C. Norris (iD), Andrew Wilson²,3 \\ ${ }^{1}$ David Norris Consulting, LLC, Seattle, WA, 98102-5384, USA \\ ${ }^{2}$ College of Nursing, University of Utah Health Sciences Center, Salt Lake City, UT, 84132, USA \\ ${ }^{3}$ Anolinx LLC, Bountiful, UT, 84010, USA
}

\section{V1 First published: 27 May 2016, 5:1014 \\ https://doi.org/10.12688/f1000research.8753.1}

Latest published: 27 May 2016, 5:1014

https://doi.org/10.12688/f1000research.8753.1

\section{Abstract}

In a 2014 report on adolescent mental health outcomes in the Moving to Opportunity for Fair Housing Demonstration (MTO), Kessler et al. reported that, at 10- to 15-year follow-up, boys from households randomized to an experimental housing voucher intervention experienced 12-month prevalence of post-traumatic stress disorder (PTSD) at several times the rate of boys from control households. We reanalyze this finding here, bringing to light a PTSD outcome imputation procedure used in the original analysis, but not described in the study report. By bootstrapping with repeated draws from the frequentist sampling distribution of the imputation model used by Kessler et al., and by varying two pseudorandom number generator seeds that fed their analysis, we account for several purely statistical components of the uncertainty inherent in their imputation procedure. We also discuss other sources of uncertainty in this procedure that were not accessible to a formal reanalysis.

Keywords

mental health, housing mobility, adolescent , post traumatic stress disorder

\section{Open Peer Review \\ Approval Status \\ 1 2 \\ version 1 \\ 27 May 2016

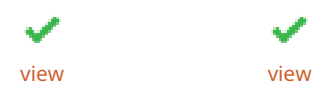 \\ 1. Ewout W. Steyerberg, Erasmus University Medical Center, Rotterdam, The Netherlands \\ 2. Fred Hasselman, Radboud University \\ Nijmegen, Nijmegen, The Netherlands \\ Any reports and responses or comments on the article can be found at the end of the article.}


Corresponding author: David C. Norris (david@precisionmethods.guru)

Competing interests: The authors have no financial, personal, or professional competing interests to declare in connection with this article.

Grant information: DCN received partial funding for travel to Salt Lake City from the University of Utah, through AW's Faculty Development Grant.

The funders had no role in study design, data collection and analysis, decision to publish, or preparation of the manuscript.

Copyright: ( 2016 Norris DC and Wilson A. This is an open access article distributed under the terms of the Creative Commons

Attribution License, which permits unrestricted use, distribution, and reproduction in any medium, provided the original work is properly cited.

How to cite this article: Norris DC and Wilson A. Early-childhood housing mobility and subsequent PTSD in adolescence: a Moving to Opportunity reanalysis [version 1; peer review: 2 approved] F1000Research 2016, 5:1014

https://doi.org/10.12688/f1000research.8753.1

First published: 27 May 2016, 5:1014 https://doi.org/10.12688/f1000research.8753.1 


\section{Introduction}

The Moving to Opportunity for Fair Housing Demonstration (MTO) was a social experiment mandated by Congress, and conducted during the 1990's. From 1994 to 1998, 4,604 households residing in distressed inner-city housing in five U.S. cities were randomized to three experimental groups. A control group received no housing voucher; a traditional voucher group received a standard 'Section 8' housing voucher; and a low-poverty voucher group received an experimental type of voucher usable only for housing located in a low-poverty area. Adults and children in the MTO households were surveyed in 2001 and 2011 to assess a variety of economic and mental health outcomes.

A 2003 MTO Interim Evaluation ${ }^{1}$ (4-7 years after randomization) revealed an interesting interaction between gender and housing mobility, with respect to outcomes of delinquency and risky behavior: girls benefited, but boys did not. This finding, somewhat at odds with earlier research ${ }^{2}$, spurred subsequent explanatory efforts $^{3-6}$.

In 2014, Kessler et al. ${ }^{7}$ published an analysis of the Final Youth Evaluation (10-15 years after randomization), reporting statistically significant and numerically substantial voucher effects on several psychiatric diagnoses in boys. Compared with controls, boys from low-poverty voucher households exhibited elevated 12-month prevalence of PTSD (6.2\% vs 1.9\%; OR, 3.4 [95\% CI, 1.6-7.4]) and 'conduct disorder' (6.4\% vs $2.1 \%$; OR, 3.1 [95\% CI, 1.7-5.8]). One of us (DCN) has previously suggested ${ }^{8}$ that these results are open to criticism on construct validity ${ }^{9}$ grounds. While working to define a protocol for empirically exploring this question, however, DCN learned that the PTSD outcome in 7 was imputed in a manner that invites scrutiny at the more basic level of statistical conclusion validity ${ }^{10}$.

\section{PTSD outcome imputation by Kessler et al.}

Although the report by Kessler et al. ${ }^{7}$ describes in some detail the imputation of missing covariates in the Final Youth Evaluation, it does not indicate that its PTSD outcome was in fact imputed. This imputation initially came to attention through a footnote on page 38 of 11, and was subsequently confirmed in a (Nov 2014) communication from the original authors.

The MTO Final Youth Survey employed an abridged, computerized self-administered version of the Composite International Diagnostic Interview (CIDI) ${ }^{12}$. The full version of the CIDI was used in the National Comorbidity Survey Adolescent Supplement (NCS-A), where its PTSD diagnostic algorithm was found to have a "moderate" concordance (AUC, 0.79) with diagnoses obtained through clinical diagnostic interviews ${ }^{13}$. The abridgment of the MTO instrument prevented direct application of the CIDI diagnostic algorithms in the MTO, however. Instead, CIDI algorithmderived lifetime PTSD diagnoses in the NCS Replication survey (NCS-R) were regressed on responses to those PTSD-related questions which were retained in the MTO instrument, and the resulting (logistic) regression model was used to impute PTSD diagnoses for the MTO Final Youth Survey respondents. (In addition to the retained PTSD-related questions, the 'obligatory' variables age, sex and race were also included as regressors; see Table 1).
Imputation was performed by Bernoulli draws consistent with the (logit) probabilities yielded by this regression equation. Responses to questions about recency of symptoms were in turn used to compute 12-month prevalence diagnoses from these imputed lifetime diagnoses.

\section{Objections to this imputation procedure}

A number of objections to this outcome imputation procedure can be articulated without recourse to a reanalysis.

The imputation is superfluous. Viewed as a link in the chain of the analysis, the imputation itself appears superfluous. It introduces an information-destroying and noise-generating transformation of the predictive model's real-valued outputs-logit probabilities on the continuous interval $(-\infty, \infty)$-to pseudorandom 'outcomes' in the set $\{n o, y e s\}$. In order to interpret conclusions about these imputed outcomes as if they were conclusions about real PTSD, it would be necessary to defend the outputs of the predictive model as genuine probabilities. But if these were true probabilities, then they could be analyzed directly, without interposing a noisy and informationdestroying pseudorandom number generation (RNG) step.

Table 1. Coefficients of the logistic regression model used to impute PTSD outcomes in Kessler et al. ${ }^{7}$.

\begin{tabular}{|c|l|}
\hline Beta & Independent variable \\
\hline-1.515 & Intercept \\
\hline 0.0263 & Age \\
\hline 0.1105 & Sex is female ( $=$ Male, $1=$ Female) \\
\hline-0.0819 & Race is hispanic \\
\hline-0.5597 & Race is black \\
\hline-0.9751 & Race is other \\
\hline-0.5603 & as child, badly beaten by parent/caregiver \\
\hline 0.0504 & badly beaten by spouse/romantic partner \\
\hline-0.3877 & badly beaten by anyone else \\
\hline 0.1148 & mugged/held up/threatened with weapon \\
\hline-0.1614 & ever raped (penetration occurred) \\
\hline 0.5993 & ever sexually assaulted or molested \\
\hline 0.078 & someone close died unexpectedly \\
\hline 0.4687 & anyone close had extreme traumatic exper. \\
\hline 0.4591 & as child, witnessed serious physical fights \\
\hline 0.1683 & saw person badly injured/killed/dead body \\
\hline-0.2237 & ever experienced other very traumatic event \\
\hline 0.3664 & purposely stay away things remind event \\
\hline-0.0581 & lose interest in things used to enjoy \\
\hline 0.2516 & feel emotionally distant/cut-off from people \\
\hline 0.1159 & trouble feeling love/happiness toward others \\
\hline 0.64 & feel no reason to plan for the future \\
\hline 0.8654 & trouble falling asleep during random event \\
\hline 0.1323 & more easily startled by ordinary noises \\
\hline 0.04
\end{tabular}


It makes the reported CIs strictly artifactual. Because a logistic regression model has no error term, the confidence intervals reported for the logistic regression of imputed PTSD on voucher treatment in Kessler et al. ${ }^{7}$ convey only the uncertainty arising from the RNG sampling performed to impute the PTSD outcomes. All of the substantive sources of uncertainty lurk in the specification and estimation of the imputation model, procedures that are opaque to a reader of 7 .

The model specification appears desultory. The specification of this outcome-imputation model gives every impression that it was regarded as purely phenomenological, and that it was specified, estimated and checked in the desultory manner customary for imputation of missing covariates. The model shows no evidence of an attempt to include additional predictors (beyond the 'obligatory' age/sex/race) that the MTO Final Youth Survey shared with NCS-R. No bootstrap-validation is described, nor is any other investigation of the predictive performance of the model in the NCS-R population against which it was estimated. No shrinkage was applied to correct for overfitting ${ }^{14}$. The model coefficients themselves (see Table 1) seem uninterpretable in causal terms, unless it can be supposed (e.g.) that being badly beaten in childhood confers protection from PTSD in nearly equal measure to the PTSD risk incurred from a sexual assault/molestation.

Generalizability of NCS-R to MTO is doubtful. Even if the use of an outcomes-imputation model had been described in 7 , and if its performance had been rigorously investigated and overfitting addressed, the question of generalizability from NCS-R to MTO populations would still remain open. Most plainly, the NCS-R and MTO Youth populations barely overlap in age (Figure 1). Also important may be the qualitative differences expected between the general-population sample of NCS-R and the inner-city MTO Youth sample, with regard to their traumatic exposures and their sources of resilience relevant to PTSD.

\section{Methods}

To develop a focused critique in an objective form, we undertook a reproduction and reanalysis of the PTSD findings in 7.

In an August 2015 communication to the original authors, we committed to principles of reanalysis articulated by Christakis and Zimmerman ${ }^{15}$. Of greatest importance, to avoid a "statistical fishing expedition" 15 , we committed to limiting the scope and methods of our reanalysis to those discussed in an earlier communication (Nov 2014), which is basically recapitulated in points 1-4 above. Our commitment incorporated an explicit exclusion of multiple aspects of the original analysis: "We do not intend to critique your multiple imputation of partially missing covariates, nor the weighting techniques you employed, nor your case-level imputation to correct for non-response. We will take these as givens, as manifested in your SAS code and in the precomputed weights in the data" (Aug 2015). Additionally, regarding as too weak Christakis and Zimmerman's requirement that original authors "should be provided with the opportunity to review and comment on the reanalysis before its acceptance for publication" 15 , we committed in this same

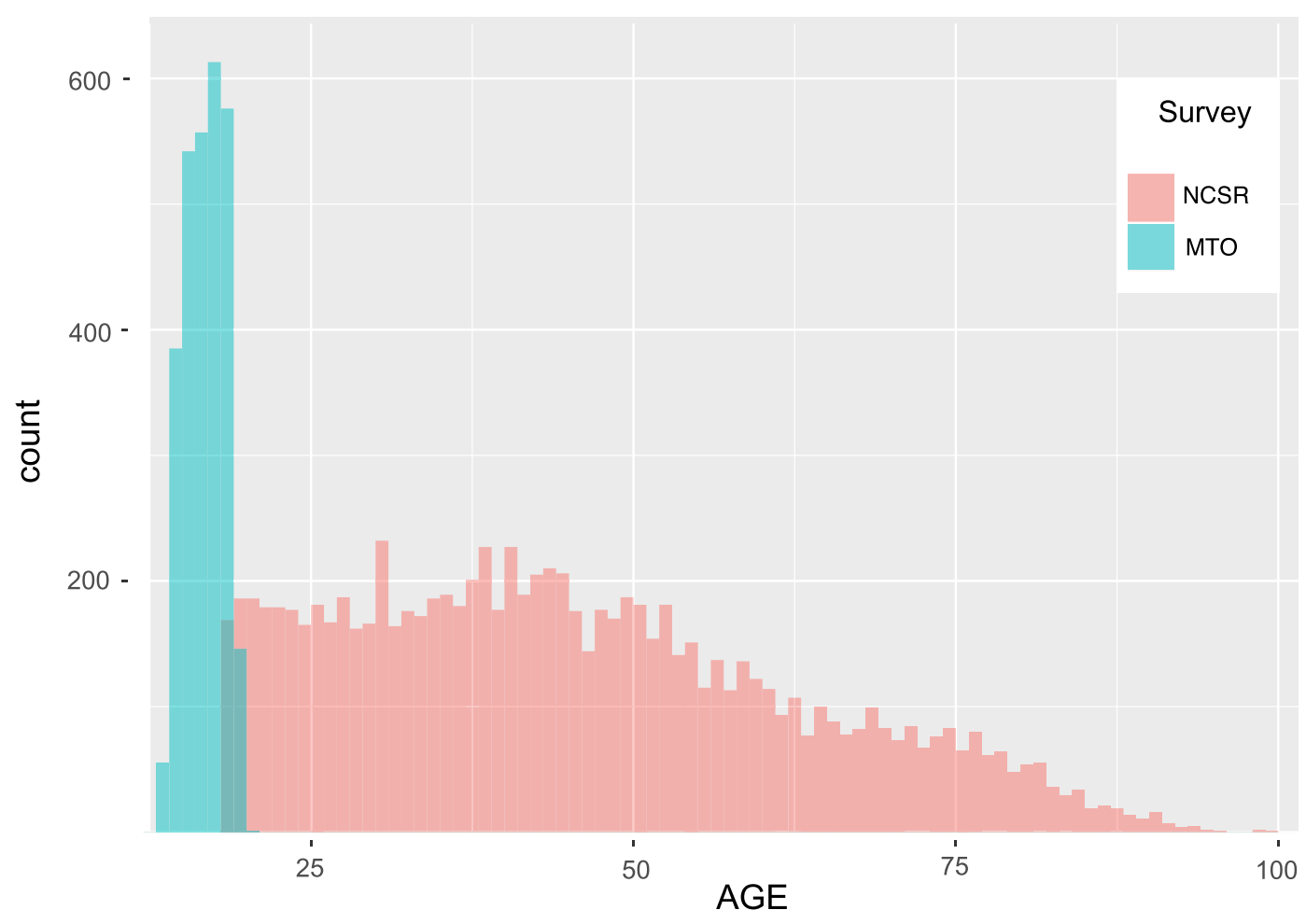

Figure 1. Age distributions of the NCS-R and MTO Youth surveys. The PTSD imputation model used in 7 was estimated in the former population, and applied to the latter. 
communication also to giving the original authors "access to all of our reanalysis code no later than our manuscript is submitted for peer review." (Said access was provided on May 3, 2016.) Finally, to avoid publication bias, authors committed to "make all reasonable efforts to publish a manuscript describing our reanalysis findings regardless of the 'significance level' of the widened confidence intervals it ultimately yields."

Our analytic code and results were maintained in a repository on GitHub, with the primary intent to support reproduction and scrutiny of our reanalysis by the original authors, as well as by reviewers and other third parties. All statistical code provided by the original researchers was checked-in to this repository exactly as received, so that any subsequent modification made by us could be readily inspected via the $g$ it diff command. A README file in the root directory of this repository provides orientation to directory structure and repository content. A single SAS script was developed which reproduces all steps of our reanalysis.

- We resampled the coefficients of the PTSD imputation model from a multivariate normal distribution with mean and covariance matrix as provided by the original authors in a Jan 2016 communication. The first 'sample' was replaced by the coefficients in Table 1 , so as to embed a formal reproduction of the original effect estimates within the larger bootstrapping exercise.

- We varied the seed used in generating the pseudorandom $U(0,1)$ probability thresholds necessary for imputing a binary PTSD 'outcome' from the (logit) probabilities produced by the PTSD imputation model. In 7, this seed was set to 1234567; we allowed this 'pr_seed' to range over $\{123,1234,12345,123456,12345 \overline{6} 7\}$.

- We varied the seed used for multiple imputation of missing covariates. In 7, the seed value was 524232; we allowed this 'mi_seed' to range over the 10 consecutive values 524230-524239.

To explore also the impact of arbitrary PTSD model specification, we performed a similar bootstrapping exercise where, instead of resampling the model coefficients, we explored a small set of alternate specifications of the imputation model. A $2 \times 2 \times 2$ grid of models was explored in which: (1) age was or was not included; (2) race was or was not included; and (3) the NCS-R sample was or was not restricted to age $\leq 40$ before estimating the model coefficients. The eight models thus produced were designated as in Table 2; model specification a1r1s99 accords with that used in 7 .

Because the standard MTO data package maintained by the Department of Housing and Urban Development (HUD) contains pre-imputed data, whereas the original analysis in 7 employed multiple imputation of the missing values in raw data, a specially prepared MTO data package was required for this work. The National Bureau of Economic Research (NBER) kindly prepared and archived this package with HUD.
Table 2. Alternative specifications explored for the logistic regression model used to impute PTSD outcomes in Kessler et $\mathbf{a} \boldsymbol{l}$. Model 'a1r1s99' is the original specification.

\begin{tabular}{|c|c|c|c|}
\hline Model & age & race & NCS-R age subset \\
\hline a0r0s40 & - & - & $\leq 40$ \\
\hline a0r0s99 & - & - & all \\
\hline a0r1s40 & - & included & $\leq 40$ \\
\hline a0r1s99 & - & included & all \\
\hline a1r0s40 & included & - & $\leq 40$ \\
\hline a1r0s99 & included & - & all \\
\hline a1r1s40 & included & included & $\leq 40$ \\
\hline a1r1s99 & included & included & all \\
\hline
\end{tabular}

\section{Results}

We reproduced the originally reported odds ratios and 95\% confidence intervals for voucher effects on 12-month prevalence of PTSD in boys: 3.44025 [1.60147-7.39026] and 2.67817 [1.23268-5.81873] for the low-poverty and traditional vouchers, respectively. (These figures were reported to 1 decimal place in the original article.)

In the course of achieving this reproduction, several unexpected features of the original analysis came to light: (1) the imputation of PTSD outcomes preceded the $20 \times$ multiple imputation of missing covariates; (2) $24.5 \%$ (456/1863) of the MTO boys were uninterviewed, and so contributed little more than their baseline characteristics to the analysis.

Figure 2 shows our $10 \times 5 \times 10=500$ bootstrapped estimates of the low-poverty voucher effect on 12-month PTSD prevalence in MTO boys, placing the original estimates (shown in red) in context.

Figure 3 shows our $8 \times 5 \times 10=400$ estimates of the low-poverty voucher effect on 12-month PTSD prevalence in MTO boys, bootstrapped over alternative specifications of the PTSD imputation model.

Each iteration of our bootstrap required just over 4 minutes on a modern Windows workstation; Figure 2 and Figure 3 thus represent about 35 and 28 hours of computation, respectively.

A public fork of our code repository may be accessed at https:// github.com/DNC-LLC/MTOpublic.

\section{Discussion}

A social science research enterprise as massive as the MTO Demonstration necessarily involves manifold layers of analysis that may obscure the provenance of analytical results and of associated 


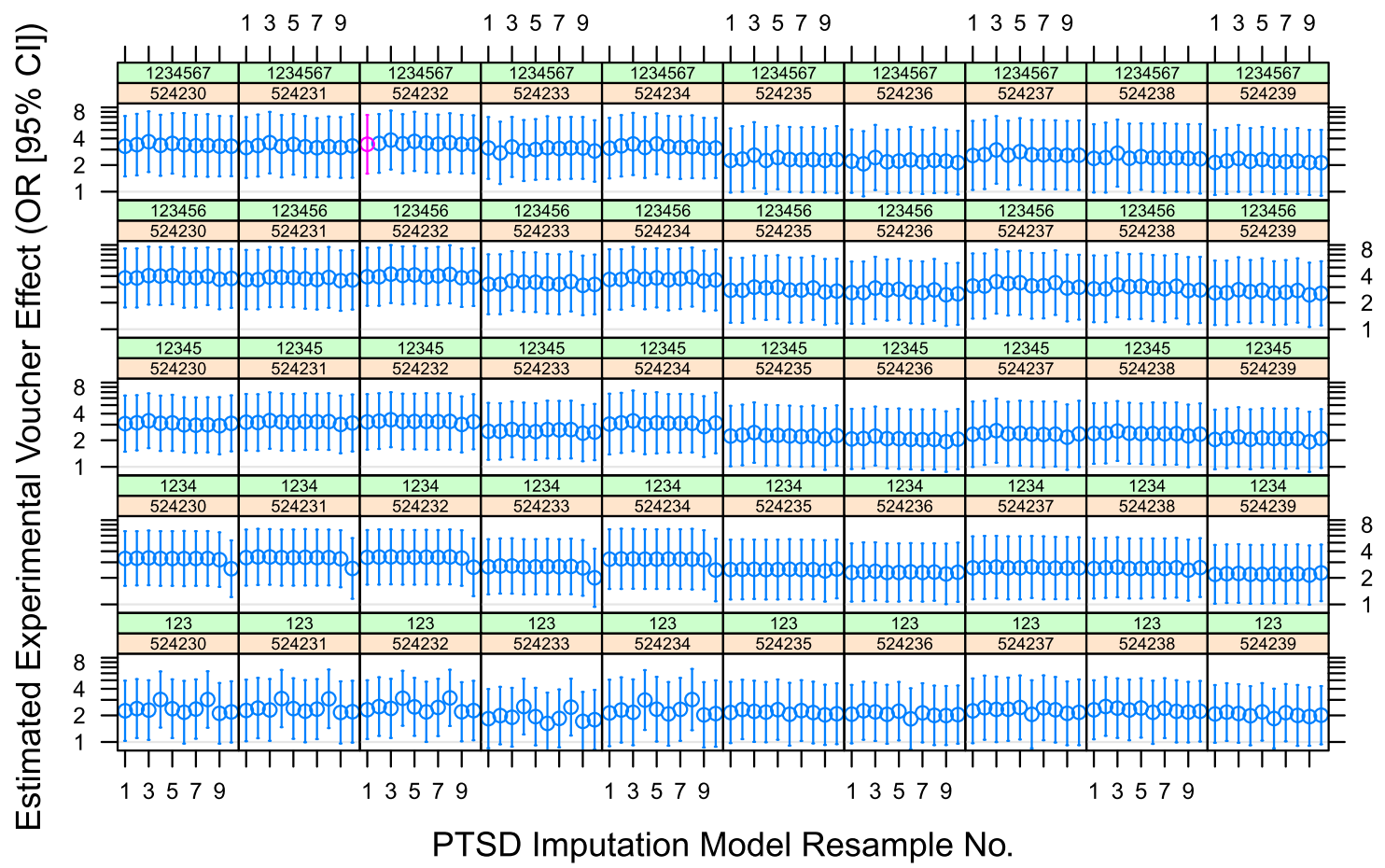

Figure 2. Bootstrapped estimates of the low-poverty voucher effect on PTSD 12-month prevalence in MTO boys. Each panel contains 10 effect estimates obtained by resampling the PTSD imputation model as described in the text, with the first 'resample' being the original coefficients as in Table 1. For each panel, the green strip shows the RNG seed used to generate pseudorandom U(0,1) thresholds for PTSD imputation, and the orange strip shows the RNG seed used for the multiple imputation of missing covariates. In 7, these seeds were set to 1234567 and 524232, respectively; thus, the effect drawn in red represents our reproduction of the published effect estimate. Note the logarithmic scale.

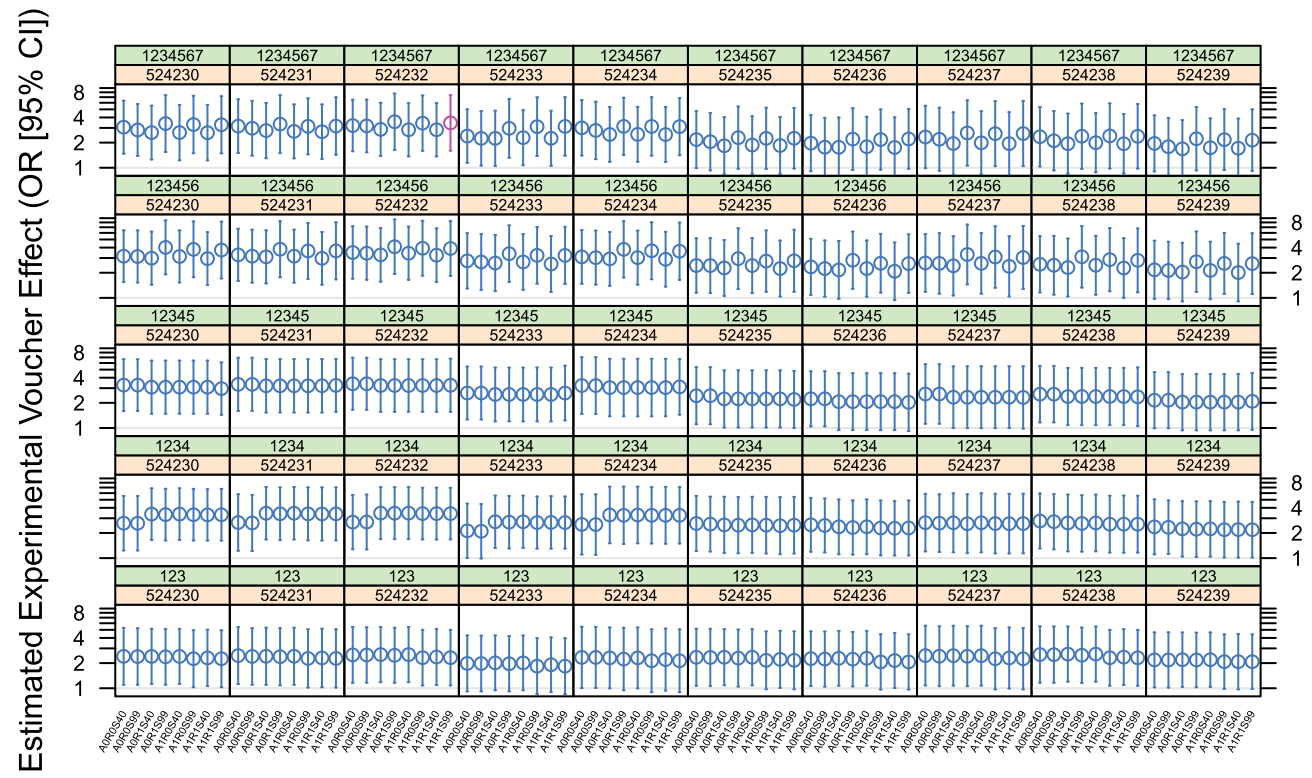

\section{PTSD Imputation Model Specification}

Figure 3. Bootstrapped estimates of the low-poverty voucher effect on PTSD 12-month prevalence in MTO boys. Each panel contains eight effect estimates obtained using the alternative PTSD imputation model specifications in Table 2. For each panel, the green strip shows the RNG seed used to generate pseudorandom $U(0,1)$ thresholds for PTSD imputation, and the orange strip shows the RNG seed used for the multiple imputation of missing covariates. In 7, these seeds were set to 1234567 and 524232 , respectively; thus, the effect drawn in red represents our reproduction of the published effect estimate. Note the logarithmic scale. 
scientific 'findings'. This situation creates a compelling rationale for reproduction and reanalysis of such research. The work presented here is, we believe, the first independent attempt to reproduce or reanalyze published results from MTO. (HUD informed us that our March 5, 2015 application for an MTO data license was the first such request received by them.)

A frequentist confidence interval should be understood as abstracting away the arbitrariness immanent in a point estimate, by situating that estimate within the context of an ensemble of imagined 'possible replications'. Regrettably, in much current statistical practice only the arbitrariness of a sampling procedure typically is recognized and objectively accounted for 16 . Yet other forms of arbitrariness, such as overfitting and model selection, are equally amenable to similar 'contextualization' by bootstrapping techniques such as we employ here. (Indeed, we have structured our reanalysis so as to accentuate this frequentist abstracting-andcontextualizing idiom; our Figure 2 and Figure 3 present a direct visual analogue of the ensemble, which we hope will prove broadly accessible to social scientists. A reanalysis performed strictly for a professional statistical audience would dispense with explicit seed variation, instead simply increasing the number of multiple imputations performed from 20 to 1000; it would likewise have done away entirely with the imputation of a binary PTSD outcome, substituting a direct analysis of the PTSD probabilities.)

The confidence intervals reported in 7 account only for the sampling inherent in the MTO design. Our bootstrapping analysis further abstracts away (1) arbitrariness arising when Kessler et al. fixed their PTSD imputation model at its maximum-likelihood estimate as if known with infinite precision; (2) the arbitrariness of what appears to have been a desultory model specification; (3) some purely technical forms of arbitrariness appearing in the form of sensitivity to RNG seed selection. While we have achieved only partial abstraction of these forms of arbitrariness, and have not addressed overfitting, it does appear that the original claim of 'statistical significance' for a voucher-on-PTSD effect in MTO boys withstands the formal challenge of our reanalysis as planned. That is to say, a randomly selected confidence interval from Figure 2 or Figure 3 typically sits above the $O R=1$ threshold.

What is clear enough, however, is that a statistical analysis that randomly selects its 'findings' from Figure 2 or Figure 3 cannot be recommended for its wholesome frequentist properties. Indeed, the evident clustering of variance within certain (combinations of) seed choices disallows any straightforward attempt to compute a single, wider confidence interval from these bootstrap samples.

The main contribution of our reanalysis may be simply to bring enough transparency to an otherwise obscure PTSD imputation procedure (1) to avert its application in further research, and (2) to encourage exploration of alternative modes of analysis of the interesting and important social-scientific and policy questions surrounding the MTO boys' exposures-and responses- to violence.

The close scrutiny involved in a reanalysis of this kind inevitably brings to light aspects of the original analysis which could not have been anticipated, provoking questions not pre-specified as part of the reanalysis. We are now interested to know the statistical impact of including in the original analysis ${ }^{7}$ (and likewise in the present reanalysis) 456 uninterviewed MTO boys for whom little more than baseline covariates were available-especially in light of the fact that the PTSD imputation preceded the multiple imputation of missing covariates. We hope to address this question in a subsequent analysis.

\section{Conclusions}

We have reproduced and reanalyzed a key finding reported by Kessler et al. ${ }^{7}$, following sound principles of reanalysis ${ }^{15}$ with particular attention to limiting our scope and methodology to issues and techniques proposed before we examined the data. The original claim of a statistically significant effect of the low-poverty voucher on PTSD in adolescent boys has formally withstood the objective challenge presented by this reanalysis. Our reanalysis also brings new transparency to outcomes-imputation methods employed in 7 , which may contribute usefully to the evaluation of this work by researchers and policy analysts.

\section{Data availability}

Open Science Framework: Dataset: Early-childhood housing mobility and subsequent PTSD in adolescence: a Moving to Opportunity reanalysis, doi 10.17605/osf.io/jepyn ${ }^{17}$

\section{Author contributions}

DCN developed the concept and design of the reanalysis, supervised the study, and drafted the manuscript. AW revised the manuscript for important intellectual content and supervised the study's secure computing environment. Both authors contributed equally to the statistical programming, data analysis and interpretation.

\section{Competing interests}

The authors have no financial, personal, or professional competing interests to declare in connection with this article.

\section{Grant information}

DCN received partial funding for travel to Salt Lake City from the University of Utah, through AW's Faculty Development Grant.

The funders had no role in study design, data collection and analysis, decision to publish, or preparation of the manuscript.

\section{Acknowledgments}

We wish to acknowledge Nicole Bailey of Anolinx, for her assistance with data licensing and acquisition, and for drawing our attention to the difference in age distributions illustrated in Figure 1; Dr. Ronald C. Kessler and his team for their kind cooperation with this reanalysis, very special thanks being due to Nancy A. Sampson for her many timely and helpful communications with us particularly regarding gaps in the public versions of NCS-R and MTO data; Matt Sciandra of NBER for preparing the special package of data and SAS code which proved essential for our reproduction and reanalysis; Anolinx for providing the secure workstation on which this work was done. 
1. Orr L, Feins JD, Jacob R, et al:: Moving to Opportunity for Fair Housing Demonstration Program: Interim Impacts Evaluation. Technical report, U.S. Department of Housing \& Urban Development, Office of Policy Development \& Research, Washington, DC, 2003.

Reference Source

2. Katz LF, Kling JR, Liebman JB: Moving to Opportunity in Boston: Early Results of a Randomized Mobility Experiment. Q J Econ. 2001; 116(2): 607-654. Publisher Full Text

3. Kling JR, Liebman JB, Katz LF: Experimental Analysis of Neighborhood Effects. Econometrica. 2007; 75(1): 83-119.

Publisher Full Text

4. Popkin SJ, Leventhal T, Weissman G: Girls in the 'Hood: The Importance of Feeling Safe. Brief 1, The Urban Institute, Washington, DC, 2008. Reference Source

5. Jackson L, Langille L, Lyons R, et al:: Does moving from a high-poverty to lowerpoverty neighborhood improve mental health? A realist review of 'Moving to Opportunity'. Health Place. 2009; 15(4): 961-970.

PubMed Abstract | Publisher Full Text

6. Clampet-Lundquist S, Kling JR, Edin K, et al.: Moving teenagers out of high-risk neighborhoods: how girls fare better than boys. Am J Sociol. 2011; 116(4): 1154-1189.

PubMed Abstract | Publisher Full Text

7. Kessler RC, Duncan GJ, Gennetian LA, et al:: Associations of housing mobility interventions for children in high-poverty neighborhoods with subsequent mental disorders during adolescence. JAMA. 2014; 311(9): 937-948.

PubMed Abstract | Publisher Full Text | Free Full Text

8. Norris DC: Housing mobility and adolescent mental health. JAMA. 2014; 312(2): 190. PubMed Abstract | Publisher Full Text

9. Cronbach LJ, Meehl PE: Construct validity in psychological tests. Psychol Bull.
1955; 52(4): 281-302.

PubMed Abstract | Publisher Full Text

10. Shadish WR, Cook TD, Campbell DT: Experimental and quasi-experimenta designs for generalized causal inference. Houghton Mifflin, Boston, 2001. Reference Source

11. Measuring DSM-IV mental disorders in the Moving To Opportunity Final Evaluation 11/26/2013. Accessed 4/21/2016.

Reference Source

12. Kessler RC, Ustün TB: The World Mental Health (WMH) Survey Initiative Version of the World Health Organization (WHO) Composite International Diagnostic Interview (CIDI). Int J Methods Psychiatr Res. 2004; 13(2): 93-121. Publisher Full Text

13. Kessler RC, Avenevoli S, Green J, et al:: National comorbidity survey replication adolescent supplement (NCS-A): III. Concordance of DSM-IV/CIDI diagnoses with clinical reassessments. J Am Acad Child Adolesc Psychiatry. 2009; 48(4): 386-399.

PubMed Abstract | Publisher Full Text | Free Full Text

14. Steyerberg EW: Clinical Prediction Models. Statistics for Biology and Health. Springer New York, New York, NY, 2009. Publisher Full Text

15. Christakis DA, Zimmerman FJ: Rethinking reanalysis. JAMA. 2013; 310(23): 2499-2500. PubMed Abstract | Publisher Full Text

16. Greenland S: Multiple-bias modelling for analysis of observational data. J R Stat Soc A. 2005; 168(2): 267-306. Publisher Full Text

17. Norris D, Wilson A: Dataset: Early-childhood housing mobility and subsequent PTSD in adolescence: a Moving to Opportunity reanalysis. Open Science Framework. 2016.

Data Source 


\section{Open Peer Review}

\section{Current Peer Review Status:}

\section{Version 1}

Reviewer Report 15 July 2016

https://doi.org/10.5256/f1000research.9420.r14561

(C) 2016 Hasselman F. This is an open access peer review report distributed under the terms of the Creative Commons Attribution License, which permits unrestricted use, distribution, and reproduction in any medium, provided the original work is properly cited.

\section{Fred Hasselman}

Learning and Plasticity, Behavioral Science Institute, Radboud University Nijmegen, Nijmegen, The Netherlands

This article discusses a re-analysis and subsequent re-interpretation of a previously published study on the important topic of PTSD in adolescence. The authors argue the imputation method used in the original article is flawed and I agree with their objections.

The scientific method is supposed to to be self-correcting; the lucid problem analysis and subsequent solutions presented in this article and decision to disseminate it through a platform like F1000Research are examples of "open science" practices that will enable us to achieve this goal for our discipline.

Competing Interests: No competing interests were disclosed.

I confirm that I have read this submission and believe that I have an appropriate level of expertise to confirm that it is of an acceptable scientific standard.

Reviewer Report 24 June 2016

https://doi.org/10.5256/f1000research.9420.r14046

(C) 2016 Steyerberg E. This is an open access peer review report distributed under the terms of the Creative Commons Attribution License, which permits unrestricted use, distribution, and reproduction in any medium, provided the original work is properly cited.

\section{Ewout W. Steyerberg}

Department of Public Health, Erasmus University Medical Center, Rotterdam, The Netherlands

This is a very timely paper in this era of debate on scientific integrity and transparency. The paper 
is a good illustration of what can be achieved by data sharing and rigorous re-analysis.

Specifically, the issues of dealing with missing data are relevant, and especially outcomesimputation methods. Imputation of missing outcome is anyway controversial; Von Hippel proposed a Multiple Imputation then Deletion (MID) approach, which has some appeal but was not supported by a recent study, which argued that auxiliary variables could be beneficial in this situation to impute missing Y (Sullivan, 2015). The Von Hippel and Sullivan papers need to be discussed briefly as educational efforts in the current paper, in line with the paragraph: The imputation is superfluous.

There is 2 main issues in the specific hypothesis studied related to housing vouchers: effect estimate and uncertainty in the effect.

1. The MTO project had a strong design, with randomization to 3 groups for evaluation of the intervention. The specific outcomes highlighted here are psychiatric diagnoses, among which PTSD; this was one of many outcomes evaluated perhaps, and not the primary outcome of this study. This brings in a multiple comparisons problem, which may be emphasized more by the current authors.

We now focus on one association that stood out among many associations that were probably evaluated. First we may wonder whether we should adjust formally for multiple testing, such as a classical Bonferroni correction for the p-value. Second, we know that the strength of the association will be overestimated, even if a true association.

The suggested boys vs girls interaction further complicates the multiple comparison issue; essentially all analyses are doubled. I would like some further discussion on these broader issues of interpreting research findings (Ioannadis, 2014).

2. Uncertainty is usually expressed as a $95 \%$ confidence interval; the current study appropriately highlights that multiple sources of uncertainty may need to be considered, specifically in the context of multiple imputation.

Overall, this paper is an interesting contribution to statistical aspects on modern analysis. It illustrates that different researchers may use different methods and approaches. It also shows some progress in scientific methods, e.g. the use of bootstrapping is now more feasible than some years ago due to ever increasing computational power. This type of reanalysis is to be applauded and stimulated to increase the credibility of scientific research findings.

\section{References}

1. Sullivan TR, Salter AB, Ryan P, Lee KJ: Bias and Precision of the. Am J Epidemiol. 2015; 182 (6): 528 34 PubMed Abstract | Publisher Full Text

2. Ioannidis JP: How to make more published research true.PLoS Med. 2014; 11 (10): e1001747 PubMed Abstract | Publisher Full Text

Competing Interests: No competing interests were disclosed.

I confirm that I have read this submission and believe that I have an appropriate level of expertise to confirm that it is of an acceptable scientific standard. 
The benefits of publishing with F1000Research:

- Your article is published within days, with no editorial bias

- You can publish traditional articles, null/negative results, case reports, data notes and more

- The peer review process is transparent and collaborative

- Your article is indexed in PubMed after passing peer review

- Dedicated customer support at every stage

For pre-submission enquiries, contact research@f1000.com 